\title{
BMJ Global Health Innovative demand creation strategies to increase voluntary medical male circumcision uptake: a pragmatic randomised controlled trial in Zimbabwe
}

Webster Mavhu (D) ,1,2 Melissa Neuman (D) , ${ }^{3}$ Karin Hatzold (D) , ${ }^{4}$ Stephen Buzuzi, ${ }^{1}$ Galven Maringwa, ${ }^{1}$ Sungai T Chabata (1) , ${ }^{1}$ Collin Mangenah (1) , Noah Taruberekera, ${ }^{5}$ Ngonidzashe Madidi, ${ }^{5}$ Malvern Munjoma, ${ }^{5}$ Getrude Ncube, ${ }^{6}$ Sinokuthemba Xaba, ${ }^{6}$ Owen Mugurungi, ${ }^{6}$ Cheryl C Johnson (D) , Elizabeth L Corbett (D) , ${ }^{8}$ Helen A Weiss (D) , ${ }^{3}$ Katherine Fielding (D) , Frances M Cowan (D) 1,2

To cite: Mavhu W, Neuman M, Hatzold K, et al. Innovative demand creation strategies to increase voluntary medical male circumcision uptake: a pragmatic randomised controlled trial in Zimbabwe. BMJ Global Health 2021;6:e006141. doi:10.1136/ bmjgh-2021-006141

Handling editor Seye Abimbola - Additional supplemental material is published online only. To view, please visit the journal online (http://dx.doi.org/10. 1136/bmjgh-2021-006141).

Received 27 April 2021 Accepted 16 June 2021

Check for updates

(C) World Health Organization 2023. Licensee BMJ.

For numbered affiliations see end of article.

Correspondence to Dr Webster Mavhu; webster@ceshhar.co.zw

\section{ABSTRACT}

Introduction Reaching men aged 20-35 years, the group at greatest risk of HIV, with voluntary medical male circumcision (VMMC) remains a challenge. We assessed the impact of two VMMC demand creation approaches targeting this age group in a randomised controlled trial (RCT).

Methods We conducted a $2 \times 2$ factorial RCT comparing arms with and without two interventions: (1) standard demand creation augmented by human-centred design (HCD)-informed approach; (2) standard demand creation plus offer of HIV self-testing (HIVST). Interpersonal communication (IPC) agents were the unit of randomisation. We observed implementation of demand creation over 6 months (1 May to 31 October 2018), with number of men circumcised assessed over 7 months. The primary outcome was the number of men circumcised per IPC agent using the astreated population of actual number of months each IPC agent worked. We conducted a mixed-methods process evaluation within the RCT.

Results We randomised 140 IPC agents, 35 in each arm. $132 / 140$ (94.3\%) attended study training and 105/132 $(79.5 \%)$ reached at least one client during the trial period and were included in final analysis. There was no evidence that the HCD-informed intervention increased VMMC uptake versus no $\mathrm{HCD}$-informed intervention (incident rate ratio (IRR) $0.87,95 \% \mathrm{Cl} 0.38$ to $2.02 ; \mathrm{p}=0.75$ ). Nor did offering men a HIVST kit at time of VMMC mobilisation (IRR $0.65,95 \% \mathrm{Cl} 0.28$ to $1.50 ; p=0.31$ ). Among IPC agents that reported reaching at least one man with demand creation, both the HCD-informed intervention and HIVST were deemed useful. There were some challenges with trial implementation; $<50 \%$ of IPC agents converted any men to VMMC, which undermined our ability to show an effect of demand creation and may reflect acceptability and feasibility of the interventions.

Conclusion This RCT did not show evidence of an effect of HCD-informed demand intervention or HIVST on VMMC uptake. Findings will inform future design and implementation of demand creation evaluations.

\begin{abstract}
WHAT IS ALREADY KNOWN?
$\Rightarrow$ Reaching men 20-35 years old, the age group at greatest risk of HIV, with voluntary medical male circumcision (VMMC) for HIV prevention remains a challenge.

WHAT ARE THE NEW FINDINGS?

$\Rightarrow$ Demand creation intervention informed by humancentred design (HCD, fully involves intended beneficiaries in intervention design) and HIV self-testing (HIVST) trialled here did not have an impact on VMMC uptake.

$\Rightarrow$ Suboptimal implementation of the HCD-informed and HIVST interventions may reflect feasibility of implementation in practice and reduced the power of the trial to show an impact.

\section{WHAT DO THE NEW FINDINGS IMPLY?}

$\Rightarrow$ Demand creation using HCD-informed approach and/or HIVST did not overcome all barriers to adult men's VMMC uptake.

$\Rightarrow$ Additional efforts and innovative demand creation approaches are required to overcome the opportunity costs men are likely to incur.

$\Rightarrow$ Evaluations of real-world interventions continue to be affected by real life challenges to implementation including a lack of control of the manner in which interventions are implemented.
\end{abstract}

Trial registration number PACTR201804003064160.

\section{INTRODUCTION}

Voluntary medical male circumcision (VMMC) is one of five key HIV prevention strategies aiming to reduce new HIV 
infections by $75 \%$. $^{1}$ By December 2019, nearly 27 million adolescent and adult men ( $\geq 10$ years) had been circumcised and an estimated 340000 new infections averted in 15 VMMC priority countries, including 260000 infections among men and 75000 among women (due to reduced secondary transmission from men). ${ }^{23}$ However, the majority of those circumcised were adolescent boys (10-19 years); reaching adult men aged $20-35$ years, who are the age group at greatest risk of HIV, remains a challenge. ${ }^{4-8}$ Strategies to increase VMMC demand among adult men including financial incentives, counselling or education, involvement of influencers and novel information delivery, have had mixed results. ${ }^{9}$ There have been calls for innovative and robust VMMC demand creation strategies to reach adult men. ${ }^{9-14}$

Within sub-Saharan Africa, barriers to VMMC uptake include the restricted number of service delivery sites and limited numbers of healthcare workers available to perform the procedure. ${ }^{15}$ Attrition of trained staff is an additional challenge. ${ }^{16}$ Men traditionally access healthcare less than women, and this might be further exacerbated by masculine norms that equate help-seeking with being 'weak'. ${ }^{17-19}$ Other barriers include poor perception of HIV risk ${ }^{20-22}$ and fear of: pain, surgical complications, preoperative HIV testing ${ }^{20} 2123$ and costs (including opportunity costs). ${ }^{10}{ }^{11}$ Previous research suggested that preoperative HIV testing was a significant barrier to VMMC uptake as many men reported being concerned about pre-VMMC testing at a VMMC site when deductive disclosure may occur in the event they are HIV positive. ${ }^{20}$ Researchers then hypothesised that if men self-tested and knew their status in advance of going to the VMMC centre, this would overcome their fear of testing for the first time at the VMMC site (even if it meant being tested again).$^{24}{ }^{25}$ Strategies to overcome the other barriers are required if the intervention is to be brought to desired scale.

In 2015, market research was undertaken in Zimbabwe to systematically understand the journey from initial awareness of VMMC, to undergoing the procedure and in turn becoming a 'VMMC advocate', as well as how that journey varies across types (or segments) of men. ${ }^{26-28}$ This research mapped the decision-making path men take to VMMC, identifying key triggers for demand and strategies that can be adopted to address the intend-to-action gap. It also used a hybrid psychographic-behavioural segmentation approach to profile men according to their perceptions about and orientation to VMMC. ${ }^{26-28}$ Through this market research, six 'segments' of men were identified based on their attitudes to and motivations for VMMC. The research also identified factors driving the cognitive dissonance (conflicting attitudes, beliefs or behaviours) impeding VMMC uptake among some segments of men. ${ }^{26} 28$

Based on this market research, the largest VMMC implementer in Zimbabwe (Population Services International (PSI)) prioritised three segments of men for demand creation interventions, who represented $56 \%$ of uncircumcised males aged 15-29 years in Zimbabwe at that time. The three prioritised segments were: VMMC enthusiasts (21\%, have a high potential to undergo VMMC and strong commitment already-although need to overcome some dissonance in order to proceed); neophytes (19\%, potentially enthusiastic but poor knowledge undermines their commitment-addressing this would be relatively straightforward and likely result in increased VMMC uptake) and embarrassed rejecters (16\%, potentially interested but with little commitment and knowledge, embarrassment and fears are high—so need lots of support to undergo VMMC). ${ }^{26} 28$

Drawing on market research findings, interpersonal communication (IPC) demand creation was redesigned to encompass a human-centred design (HCD)-informed approach. IPC agents who act as community mobilisers for VMMC were trained to conduct one-on-one sessions with potential VMMC clients using three tools: a segmentation typing tool, segment-specific targeted messaging and a pain-o-metre (visual aid) to guide discussions related to pain (see online supplemental appendix for detailed descriptions).

Here, we present results of a randomised controlled trial (RCT) that assessed the effectiveness of this HCD-informed approach in motivating 15-year-old to 29-year-old men (Zimbabwe's VMMC priority age group) to take up VMMC. In addition, we explored whether providing access to HIVST would result in increased VMMC uptake. We hypothesised that potential VMMC clients mobilised: (1) using the HCD-informed intervention would have increased circumcision uptake compared with those mobilised using standard techniques and (2) with the offer of HIVST would have increased circumcision uptake compared with those mobilised without the offer of HIVST.

\section{METHODS}

\section{Study design and setting}

We conducted a $2 \times 2$ factorial pragmatic RCT comparing arms with and without two interventions: (1) standard demand creation augmented by HCD-informed approach; (2) standard demand creation plus offer of HIVST.

We conducted the study in five districts (Buhera, Gokwe North, Mangwe, Mutasa, Zvimba), in 4 of 10 provinces in Zimbabwe. Neither interventions had been implemented in these districts before the trial. The interventions were implemented by IPC agents, all of whom had at least some secondary education. To guard against the risk of contamination, each IPC agent was assigned a ward (subdivision of district) and required to work within the confines of their ward.

Outcomes were measured using monthly VMMC programme outputs collected by these agents and VMMC clinics.

CONSORT guidelines for $\mathrm{RCT}^{29}$ reporting were followed. Three changes were made to the trial protocol 
after trial commencement. First, the intervention run-in period was extended to allow more time for the IPC agents to become comfortable with the HCD-informed approach. Second, following review of blinded interim data, the sample size was recalculated and the main analysis was changed from parallel comparisons to the two factorial comparisons, assessing outcomes with and without each intervention. Third, it was necessary to change the statistical model documented in the initial analysis plan to accommodate extremely high variability of outcomes between and within IPC agents; a more valid approach was used. These changes are summarised in detail in the online supplemental appendix.

For each study arm, training was conducted over 2 days from 1 to 8 February 2018 and implementation started straight after with a closely supervised run-in period from 20 February to 30 April 2018. By 30 April, implementers were confident that all IPC agents were able to implement demand creation as assigned. The interventions were implemented over 6 months ( 1 May to 31 October 2018) and counting number of circumcisions conducted over 7 months (1 May to 30 November 2018), allowing the last man enrolled on 31 October to have 1 month to be circumcised. This minimum follow-up is in keeping with the implementer's experience that men wanting to be circumcised will do so within 1 month of referral.

\section{Client involvement}

The interventions were optimised for this setting using formative work undertaken in 2017 with VMMC clients and IPC agents. For example, during the formative work, it was observed that IPC agents did not know how to categorise men who were clearly willing to be circumcised prior to segmentation. Consequently, the implementer created an additional segment ('green'-a default segment which denoted that client was willing to be circumcised before the start of the demand creation session and therefore no proper segmentation was done).

\section{Randomisation and masking}

IPC agents were the unit of randomisation and were assigned 1:1:1:1 to four arms, using restricted randomisation by IPC agent for sex, age and having $\geq 12$ months of VMMC mobilisation experience. The random allocation of IPC agents to intervention arms was completed using random-number tables generated in Stata by a study statistician on 25 January 2018.

Neither IPC agents nor clients could be masked to allocation. The statistician conducting the primary analysis was masked to allocation for the planned primary and secondary analyses. Subsequent analyses were conducted on unmasked data.

\section{Intervention components}

Arm 1: standard demand creation

IPC agents received basic training on how to promote VMMC, including identifying barriers, clarifying myths and misconceptions and summarising key benefits. Men were mobilised for VMMC individually or in groups. Clients referred for VMMC either went to VMMC sites on their own or were taken there in a programme vehicle.

\section{Arm 2: standard demand creation plus offer of HIVST}

In addition to standard demand creation, IPC agents offered the men they mobilised a HIVST kit, with a kit use demonstration if accepted.

\section{Arm 3: HCD-informed demand creation approach}

IPC agents received basic training in the HCDinformed approach, including using the segmentation typing tool, to prioritise the three key segments. They then delivered messages tailored to that 'segment'. IPC agents were specifically required to address any painrelated concerns using a visual aid (pain-o-metre) to outline the VMMC procedure, healing process, as well as possible pain management techniques. Men in the non-prioritised segments received general (as opposed to tailored) messages.

As already stated, if a client was willing to be circumcised at the start of the discussion, segmentation was skipped and these men were allocated to a default 'segment' (green), which was not developed from the market research. Clients mobilised in groups at schools were also allocated to the 'green' segment.

\section{Arm 4: HCD-informed demand creation approach plus offer of} HIVST

In this arm, in addition to the HCD-informed demand creation approach, IPC agents offered the men they mobilised a HIVST kit, with a kit use demonstration if accepted.

\section{Additional procedures-all arms}

After a mobilisation session, each client was asked to provide his contact details to allow the IPC agent to provide supportive follow-up. All men who were referred for VMMC were given a referral card with a unique identifier and asked to present it when they attended for VMMC, enabling their attendance to be linked with the referring IPC agent. District Field Officers (IPC agents' supervisors) checked concordance between IPC agents and facility records, which helped curb fraud in payments to IPCs. The RCT payment structure followed that of the national VMMC programme, with IPC agents receiving US $\$ 5$ when a boy aged $10-14$ years or a man $\geq 30$ years was circumcised and US $\$ 7$ when a man aged 15-29 years was circumcised. IPCs only received an incentive if the person they reached and referred was actually circumcised.

\section{Process evaluation}

We conducted a mixed-methods process evaluation within the trial. Data included: programme data; observations of IPC agents conducting VMMC mobilisation sessions; in-depth interviews (IDIs) and focus 


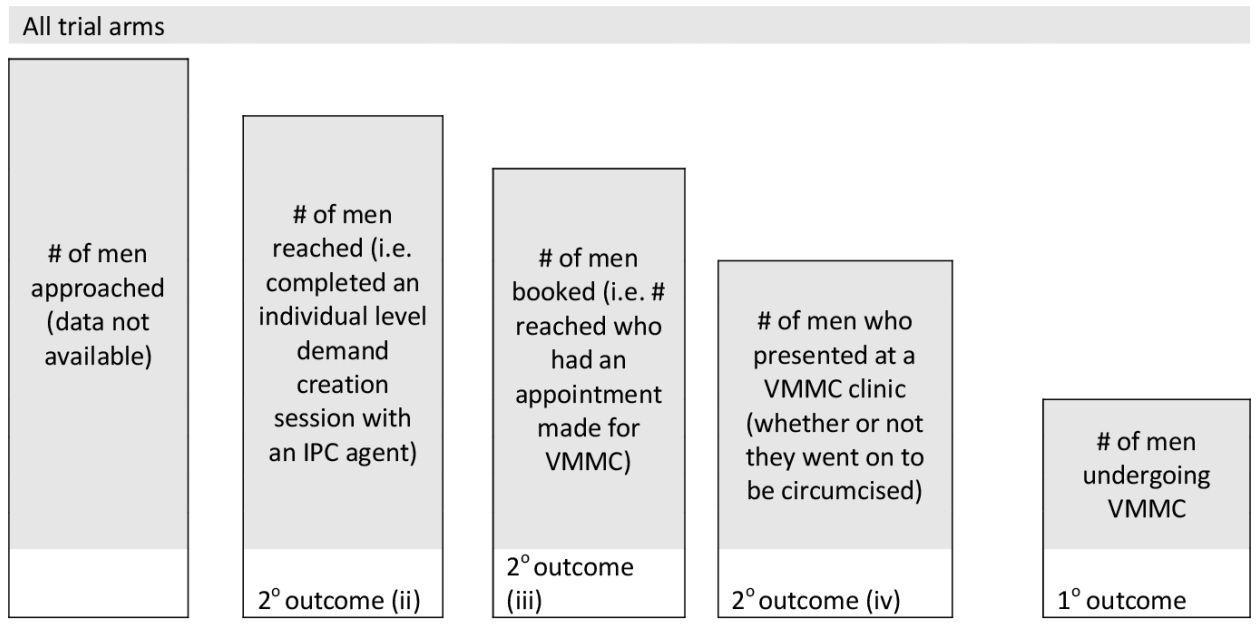

HIV ST arms only
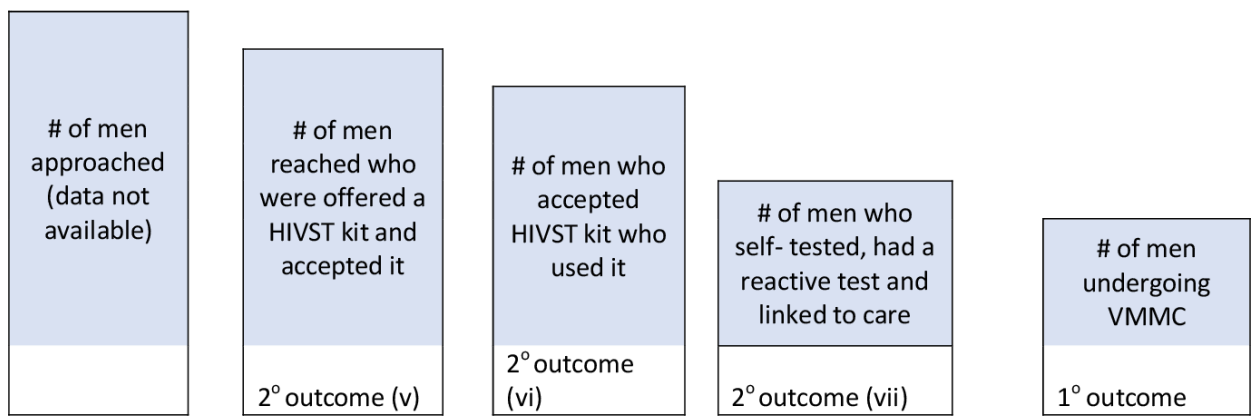

Figure 1 Primary outcome and secondary outcomes (ii)-(vii). HIVST, HIV self-testing; IPC, interpersonal communication; VMMC, voluntary medical male circumcision.

group discussions (FGDs) with IPC agents; IDIs with PSI's District Field Officers; and FGDs with men mobilised for VMMC. Iterative qualitative data collection and analysis informed a grounded thematic analytical approach.

\section{Outcome measures}

The primary outcome was the number of men circumcised per IPG agent over the 6-month duration of the trial and compared by arm. Secondary outcomes were: (i) the mean conversion proportion (conversion proportion defined as number of men circumcised divided by the number of men reached by IPC agents); secondary outcomes (ii)-(vii) are shown in figure 1 . In addition, there were three HIVST-related outcomes measured in the two HIVST arms (figure 1).

Programme data collected from IPC agents and clinic data were used to evaluate the primary outcome and secondary outcomes (i)-(iv). For ST outcomes (v)(vii), IPC agents recorded whether the man opted to take a kit. Additional data on HIVST use were obtained from a phone-administered follow-up questionnaire with clients.

\section{Sample size calculation}

As originally designed, this study required 35 IPC agents per arm (140 IPC agents across four arms) to have $80 \%$ power to detect a $30 \%$ proportionate difference in VMMC uptake between any two individual arms assuming a high variability between IPC agents (coefficient of variation, $\mathrm{k}=0.3$ ) (see online supplemental appendix for other scenarios). A revised power calculation was completed in October 2018, using a higher $\mathrm{k}$ (1.0) to reflect high measured clustering within the outcome data collected to that point. This sample size calculation found that with 65 IPC agents per arm and two arms, we had $80 \%$ power to detect between $90 \%$ increase in VMMC per intervention (eg, 10.5-20). This resulted in the change from parallel comparisons to factorial-arm comparisons, assessing outcomes with and without each intervention.

\section{Statistical analysis}

Analysis used intention-to-treat (ITT) and as-treated populations. The as-treated analysis population was based on the actual number of months each IPC agent worked, restricted to agents who reached at least one client, and included 20 IPC agents who the implementer added postrandomisation to replace 20 randomised IPC agents who declined to take part before training. The ITT analysis population was as defined based on the intended 6 months of mobilisation time each IPC agent was to work and excluded the 20 IPC agents added by the implementer postrandomisation. 
The study team and the independent Technical Advisory Group (TAG) viewed the 'as-treated' analysis as the most appropriate for understanding the real-world effectiveness of the intervention and was therefore considered the primary analysis. This analysis compared outcomes with and without each intervention (factorial-arm analysis).

A statistical analysis plan (SAP) was developed prior to analysis; however, because the prespecified modelling approach was not valid statistically, the SAP was revised both during data collection and after the initial analysis was complete with input from the TAG. For the primary outcome, the effect of study arm was assessed at the IPC agent level using negative binomial regression. Subgroup analyses were performed to assess the differences in impact of the outcome by client age. These analyses were prespecified as 15-19 years, 20-29 years and 30+ years and then an additional post hoc analysis of 18-19 year-olds.

Conversion proportion per IPC was modelled using logistic regression. Secondary outcomes (ii)-(iv) were analysed at the same IPC agent-level data using negative binomial regression, while secondary outcomes related to self-test use were analysed using logistic regression.

All analyses were repeated using parallel-arm comparisons as well as ITT and as-treated populations. HIVST outcomes were compared by study arm 2 and 4 at the client level, using data arising from the as-treated analysis population.

Analyses were done with STATA V.15.1 software (StataCorp).

\section{RESULTS}

One hundred and forty-three IPC agents were identified by PSI for the trial, 140 were randomly chosen and allocated to one of four study arms. Postrandomisation, 20 declined to take part before training and were replaced with IPC agents by the implementer. One hundred and thirty-two of 140 (94.3\%) attended study arm-specific training and 105/132 $(79.5 \%)$ reported reaching at least one client during the trial period and were included in the 'as-treated' analysis. IPC agent attrition ranged from 4 (11.8\%) to $11(33.3 \%)$ by arm and was highest in arm 4 (figure 2).

\section{Characteristics of IPC agents}

There were differences in characteristics of IPC agents by arm resulting from replacement of 20 IPCs postrandomisation and included differences in age, gender, level of education and prior VMMC mobilisation experience (table 1$)$

\section{Characteristics of clients reached}

Overall, 105 IPC agents reached 8707 men with demand creation for VMMC. Arm 1 (SOC) reached the greatest number of clients $(n=3105,35.7 \%)$ while arm $4(\mathrm{HCD}+\mathrm{ST})$ reached the smallest $(\mathrm{n}=1542$, $17.7 \%$ ) (table 2). The majority of potential VMMC clients were out of school, across all the arms (table 2). Overall, $84.5 \%(7356 / 8707)$ of men reached were aged 15-29 years.

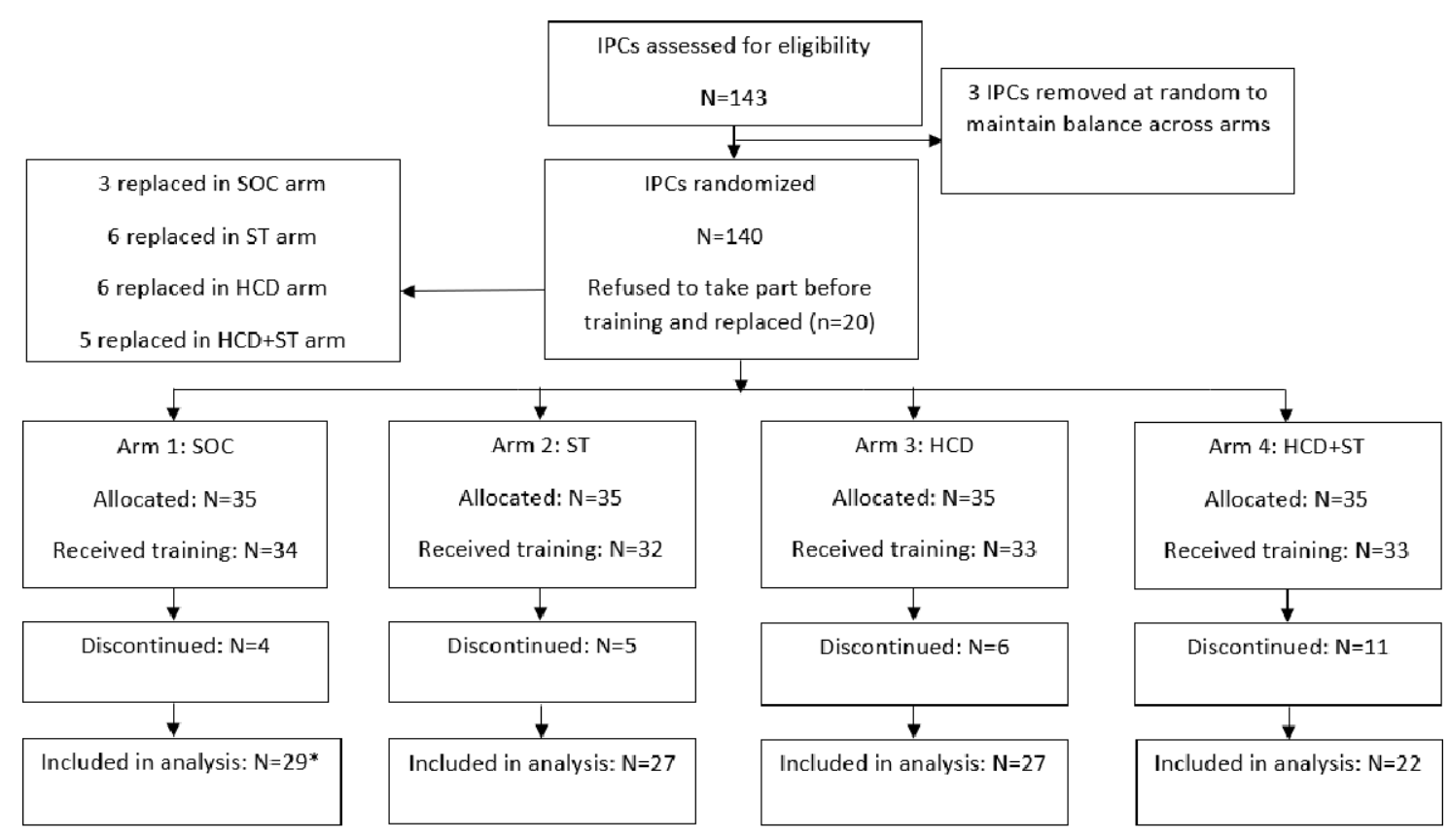

*1 IPC excluded from analysis because he only had clients circumcised after the trial period

Figure 2 CONSORT diagram for as-treated analysis population. HCD, human-centred design; IPC, interpersonal communication; SOC standard of care; ST self-testing. 
Table 1 Characteristics of IPC agents by arm (as-treated analysis population)

\begin{tabular}{|c|c|c|c|c|c|c|c|c|}
\hline & \multicolumn{2}{|c|}{ Arm 1: SOC } & \multicolumn{2}{|c|}{ Arm 2: ST } & \multicolumn{2}{|c|}{ Arm 3: HCD } & \multicolumn{2}{|c|}{ Arm 4: HCD+ST } \\
\hline & $\mathbf{n}$ & $\%$ & $\mathbf{n}$ & $\%$ & $\mathbf{n}$ & $\%$ & $\mathbf{n}$ & $\%$ \\
\hline IPC agents & 29 & & 27 & & 27 & & 22 & \\
\hline Total IPC mobilisation-months & 126 & & 96 & & 101 & & 97 & \\
\hline Male & 8 & 27.6 & 17 & 63.0 & 15 & 55.6 & 8 & 36.4 \\
\hline \multicolumn{9}{|l|}{ District } \\
\hline Buhera & 6 & 20.7 & 8 & 29.6 & 2 & 7.4 & & 9.1 \\
\hline Gokwe North & 4 & 13.8 & 9 & 33.3 & 4 & 14.8 & & 40.9 \\
\hline Mangwe & 4 & 13.8 & 1 & 3.7 & 3 & 11.1 & & 13.6 \\
\hline Mutasa & 5 & 11.1 & 3 & 11.1 & 11 & 40.7 & & 31.8 \\
\hline Zvimba & 10 & 34.5 & 6 & 22.2 & 7 & 25.9 & & 4.6 \\
\hline \multicolumn{9}{|l|}{ Age (years) } \\
\hline$\leq 19$ & 1 & 3.5 & 1 & 3.7 & 4 & 14.8 & 1 & 4.6 \\
\hline $20-29$ & 4 & 13.8 & 12 & 44.5 & 6 & 22.2 & 5 & 22.7 \\
\hline $30-39$ & 7 & 24.1 & 8 & 29.6 & 4 & 14.8 & 2 & 9.1 \\
\hline $40-49$ & 9 & 31.0 & 5 & 18.5 & 9 & 33.4 & 13 & 59.0 \\
\hline $50+$ & 8 & 27.6 & 1 & 3.7 & 4 & 14.8 & 1 & 4.6 \\
\hline \multicolumn{9}{|l|}{ Educational level } \\
\hline Primary level & 8 & 27.6 & 1 & 3.7 & 1 & 3.7 & 0 & 0 \\
\hline Secondary level & 15 & 51.7 & 23 & 85.2 & 25 & 92.6 & 22 & 100 \\
\hline$\geq$ Advanced level & 6 & 20.7 & 3 & 11.1 & 1 & 3.7 & 0 & 0 \\
\hline \multicolumn{9}{|l|}{ IPC experience } \\
\hline$<12$ months & 22 & 75.9 & 18 & 66.7 & 21 & 77.8 & 9 & 40.9 \\
\hline$\geq 12$ months & 7 & 24.1 & 9 & 33.3 & 6 & 22.2 & 3 & 59.1 \\
\hline Mean/median IPC mobilisation months & $4.2 / 4$ & & $3.7 / 2$ & & $3.8 /$ & & 4.4 & \\
\hline
\end{tabular}

HCD, human-centred design; IPC, interpersonal communication; SOC, standard of care; ST, self-testing.

Effect of HCD-informed intervention on VMMC uptake (astreated analysis population)

For the primary outcome, there was no evidence that the HCDinformed intervention increased VMMC uptake (incident rate ratio (IRR) $0.87,95 \%$ CI 0.38 to $2.02 ; \mathrm{p}=0.75$ ) (figure 3 and online supplemental table 1). There was no evidence of an interaction effect between HCD-informed intervention and age for VMMC uptake $(\mathrm{p}=0.27)$. The HCD-informed

Table 2 Characteristics of $\geq 15$-year-olds clients reached by arm (as-treated analysis population)

\begin{tabular}{|c|c|c|c|c|c|c|c|c|}
\hline & \multicolumn{2}{|c|}{ Arm 1: SOC } & \multicolumn{2}{|c|}{ Arm 2: ST } & \multicolumn{2}{|c|}{ Arm 3: HCD } & \multicolumn{2}{|c|}{ Arm 4: HCD+ST } \\
\hline & $\mathbf{n}$ & $\%$ & $\mathbf{n}$ & $\%$ & $\mathbf{n}$ & $\%$ & $\mathbf{n}$ & $\%$ \\
\hline Total & 3105 & 100 & 1754 & 100 & 2306 & 100 & 1542 & 100 \\
\hline \multicolumn{9}{|l|}{ Age in years } \\
\hline $15-17$ & 1102 & 35.5 & 592 & 33.8 & 886 & 38.4 & 509 & 33.0 \\
\hline $18-19$ & 571 & 18.4 & 318 & 18.1 & 523 & 22.7 & 278 & 18.0 \\
\hline $20-29$ & 952 & 30.7 & 440 & 25.1 & 627 & 27.2 & 558 & 36.2 \\
\hline $30+$ & 480 & 15.4 & 404 & 23.0 & 270 & 11.7 & 197 & 12.8 \\
\hline Median age (min-max), years & 19.0 & $(15-78)$ & 19.0 & $(15-87)$ & 18.0 & $(15-84)$ & 19.0 & $(15-83)$ \\
\hline \multicolumn{9}{|l|}{ Education completed* } \\
\hline School-going & 1232 & 40.1 & 638 & 39.6 & 728 & 31.8 & 477 & 31.5 \\
\hline Out of school & 1841 & 59.9 & 1041 & 60.4 & 1560 & 68.2 & 1039 & 68.5 \\
\hline
\end{tabular}

*106 missing.

HCD, human-centred design; SOC, standard of care; ST, self-testing. 


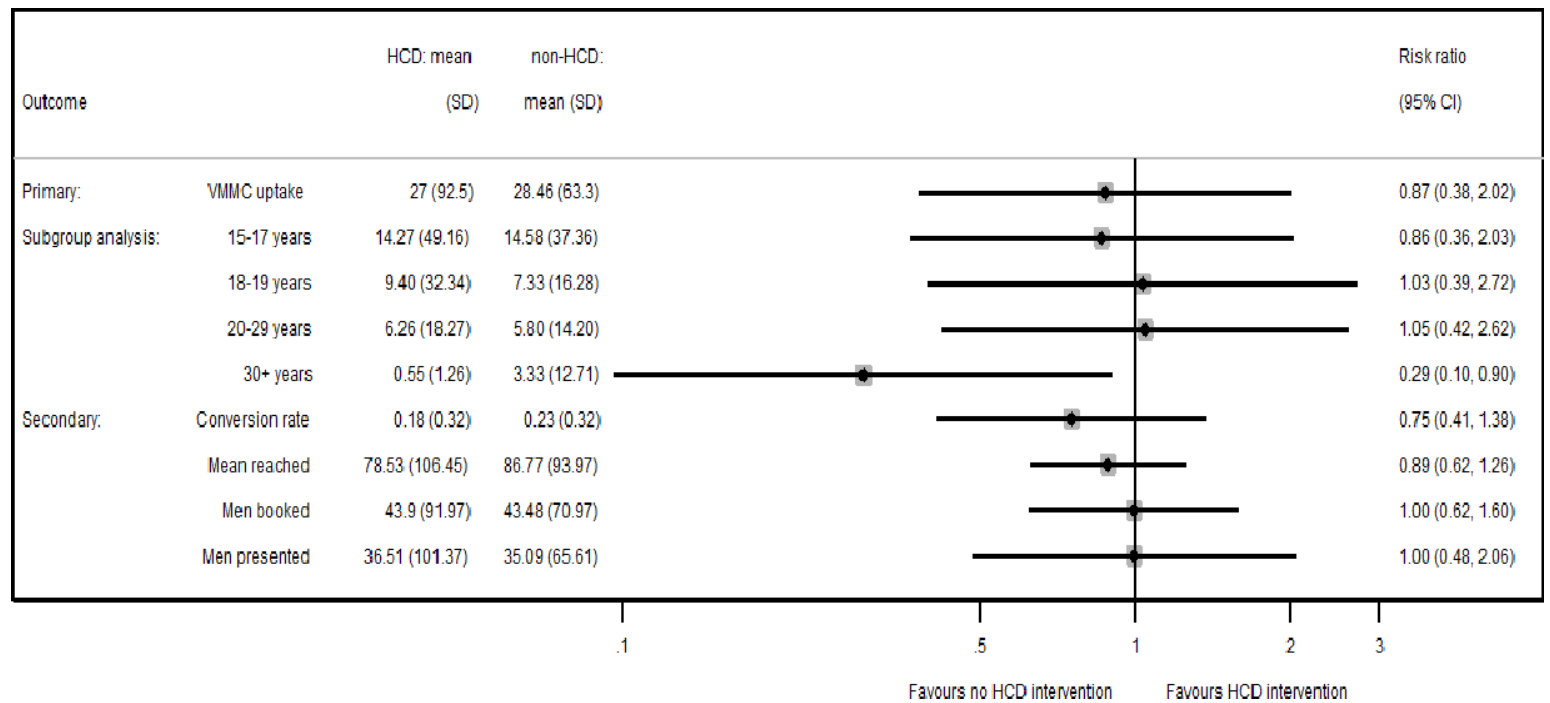

Figure 3 Effect of HCD-informed intervention-as-treated analysis population. HCD, human-centred design; VMMC, voluntary medical male circumcision.

approach had no effect on secondary outcomes of: VMMC conversion proportion (risk ratio (RR) $0.75,95 \%$ CI 0.41 to 1.38; $\mathrm{p}=0.36$ ), the number of men reached (IRR $0.89,95 \% \mathrm{CI}$ 0.62 to 1.26 ; $\mathrm{p}=0.50$ ), the number of men booked for VMMC (IRR $1.00,95 \%$ CI 0.62 to $1.60 ; \mathrm{p}>0.99$ ) or the number of men presenting for VMMC (IRR 1.00, 95\% CI 0.48 to 2.06; $\mathrm{p}>0.99)$. Overall, of all reached in the HCD arms, $12.6 \%$ (485/3848) were classified in the default 'green' segment, denoting that client was willing to be circumcised before the start of the demand creation session.

\section{Effect of HIVST intervention on VMMC uptake}

Offering men a HIVST kit at the time of VMMC mobilisation did not have an effect on VMMC uptake (IRR 0.65, 95\% CI 0.28 to $1.50 ; \mathrm{p}=0.31$ ) (figure 4 and online supplemental table 1). There was also no effect of offer of a HIVST kit on secondary outcomes of: the conversion proportion (RR 1.11, $95 \%$ CI 0.61 to 2.02; $\mathrm{p}=0.71$ ); the number of men reached (IRR $0.75,95 \%$ CI 0.53 to $1.07 ; \mathrm{p}=0.11$ ), booked (IRR 0.80 , $95 \%$ CI 0.50 to $1.29 ; \mathrm{p}=0.36$ ) or presenting for VMMC at the clinic (IRR $0.84,95 \%$ CI 0.40 to $1.73 ; p=0.63$ ). There was no evidence of an effect of an offer of HIVST on VMMC uptake in any age category although there was doubling of VMMC uptake among men over 30 years, although the CI included one (figure 4). There was no evidence of an interaction effect between HIVST intervention and age $(\mathrm{p}=0.21)$.

ITT analysis also showed no evidence of an effect of HCD-informed and HIVST approaches on VMMC uptake (see online supplemental table 2).

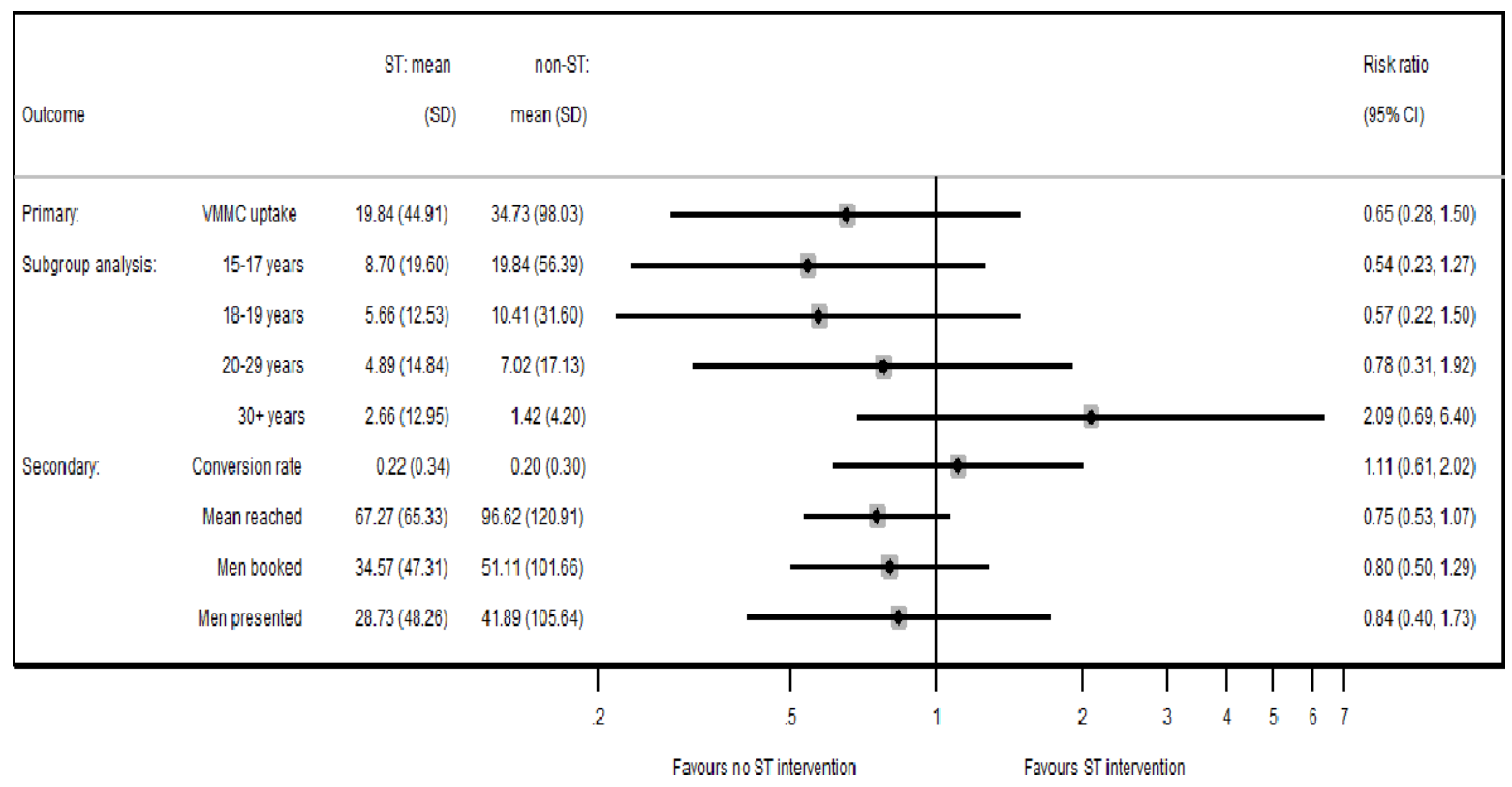

Figure 4 Effect of ST intervention-as-treated analysis population. ST, self-testing; VMMC, voluntary medical male circumcision. 
Table 3 Uptake of HIVST, by study arm (ST vs HCD+ST)

\begin{tabular}{|c|c|c|c|c|c|c|}
\hline & \multicolumn{2}{|c|}{ Arm 2: ST } & \multicolumn{2}{|c|}{ Arm 4: HCD+ST } & \multicolumn{2}{|c|}{ Effect of HCD+ST vs ST } \\
\hline & $n / N$ & $\%$ & $\mathrm{n} / \mathrm{N}$ & $\%$ & OR $(95 \% \mathrm{CI})$ & $P$ value \\
\hline Men offered HIVST (\% surveyed) & $129 / 173$ & 74.6 & $71 / 110$ & 64.6 & $0.62(0.37$ to 1.04$)$ & 0.072 \\
\hline Men accepting HIVST (\% offered) & $101 / 129$ & 78.3 & $52 / 71$ & 73.2 & 0.76 (0.39 to 1.49$)$ & 0.420 \\
\hline Men using HIVST (\% accepted) & $91 / 101$ & 90.1 & 50.52 & 96.2 & 2.75 (0.58 to 13.03$)$ & 0.203 \\
\hline Reactive HIVST (\% used) & $5 / 91$ & 5.5 & $1 / 50$ & 2.0 & $0.37(0.04$ to 3.30$)$ & $0.376(0.072)^{\star}$ \\
\hline $\begin{array}{l}\text { Men with reactive test linked to care } \\
\text { (\% reactive) }\end{array}$ & $2 / 5$ & 40.0 & $1 / 1$ & 100.0 & & \\
\hline
\end{tabular}

\section{VMMC cascade results-HCD-informed intervention segments}

Men in the three prioritised segments (embarrassed rejecters, neophytes, enthusiasts) were more likely to be circumcised than those in the non-prioritised segments. Overall however, men in the green (default) segment were much more likely than any other segment to undergo circumcision $(>70 \%$ ) (see online supplemental figure 1).

\section{HIVST secondary outcomes}

In total, 3296 men were reached by IPC agents in the HIVST arms (ST and HCD+ST arms), of whom only $8.6 \%(283 / 3296)$ were successfully followed by phone to ask questions about HIVST kit uptake and use. Overall, 200/283 (70.6\%) men reported being offered HIVST kits. There was weak evidence that participants in the $\mathrm{HCD}+\mathrm{ST}$ arm were less likely to be offered kits compared with participants in the ST arm (OR $0.62,95 \%$ CI 0.37 to 1.04; $\mathrm{p}=0.07)$. Overall, $76.5 \%$ of men who were offered a kit accepted it and 92.2\% (141/153) of those accepting reported using the kit, again with no evidence of difference between arms (table 3 ).

\section{Process evaluation findings}

\section{Acceptability of the interventions}

Most IPC agents allocated to the HCD-informed approach found it useful, noting that engaging oneon-one was more effective, resulting in more men getting quality information than was possible when using a group approach. However, all felt that some components (especially segmentation) lengthened the IPC session, resulting in fewer men 'reached' compared with using traditional IPC approaches.

Regarding HIVST, IPC agents felt that the intervention generated huge interest in the communities where HIVST had not been previously available. There was high demand for HIVST across all five districts, which appeared to be independent of intention to get circumcised.

\section{Uneven performance among IPC agents and districts}

There were substantial differences in the performance of IPC agents across all outcomes. Only 105 (79.5\%) of the 132 trained IPC agents reached at least one man to discuss VMMC over the course of the trial (and so were included in the 'as-treated' analysis) (see online supplemental table 3). Forty (38.1\%) did not convert anyone to VMMC during the trial and $30(28.6 \%)$ converted fewer than 6 men (one per month). Conversely, two IPC agents converted over 50 per month (one converted 553 over 6 months and the other converted 355).

In addition to variability between IPC agents, there was variability in implementation between study districts (conversion proportion ranging from $6 \%$ in Zvimba to $81 \%$ in Buhera), possibly due to contextual factors including number of men who were eligible to be circumcised. IPC agents in Buhera and Gokwe North devoted considerable time to VMMC mobilisation, perhaps reflecting the lack of employment opportunities locally. In the other districts, IPC agents were involved in additional income-generating activities. These factors likely explain some of the variability observed between study districts (online supplemental table 5).

\section{Implementation of the interventions}

Implementation of the HCD-informed intervention was not always as intended. As segmentation lengthened the time for mobilisation, this resulted in some IPC agents resorting to traditional approaches or administering an abridged and possibly suboptimal version of the approach. IPC agents also reported they were sometimes selective about which messages they delivered to each segment (eg, they left out messages they viewed as age and sexual experience inappropriate). Of note, IPC agent gender, age and education were significantly associated with VMMC uptake (online supplemental table 4).

The HIVST intervention was similarly not implemented as intended. As IPC agents were the only source of ST kits in the RCT districts, they did not want to be seen as 'promoting HIVST' instead of VMMC, with the result that HIVST kits were not always offered by IPC agents in HIVST arms. This was in part because IPC agents sometimes reported that they felt men taking HIVST kits were not necessarily interested in VMMC. This may have been true; an appreciable proportion of the men we were able to follow-up by phone obtained ST kits and reportedly 
tested but did not take up VMMC. Further, ST kits were more likely to be offered in arm 2 (HIVST arm) than in arm 4 (HCD and HIVST), where the intervention took longest to deliver and which consequently had the highest data burden. IPC agents also found it difficult to incorporate the demonstration of the HIVST kit or the demonstration video into the IPC session. Of note, IPC agents received an incentive if their clients were circumcised but not if they self-tested, which may also have reduced their enthusiasm for HIVST distribution and demonstration.

\section{DISCUSSION}

We conducted a pragmatic randomised $2 \times 2$ factorial trial to determine the effectiveness of the HCD-informed approach and HIVST in motivating men (15-29 years) to take up VMMC. Our trial found no evidence that HCDinformed mobilisation or the offer of a HIVST kit prior to VMMC had an effect on VMMC uptake or on secondary outcomes.

The lack of detectable effect may be due to a number of factors. First, the market research conducted in 2015 identified several barriers or facilitators to VMMC uptake and recommended a set of interventions be delivered through multiple channels. ${ }^{26-28}$ However, due to resource constraints, these multiple approaches were not feasible to implement in practice. The HCDinformed approach tested here only addressed a subset of barriers. For example, we know that fear of financial loss was not addressed and this could be an especially important barrier in settings where majority of men are self-employed. Recent systematic reviews ${ }^{9} 30$ have found that financial incentives framed as fair compensation (rather than as lottery) appear to be the most acceptable and effective VMMC demand creation intervention found to date especially among adult men.

As stated earlier, men who were already deemed by IPC agents in HCD-informed approach arms to be highly motivated to VMMC and did not require the use of the segmentation typing tool were allocated to the 'green' segment. They had the highest rate of conversion to VMMC. We do not know what segment they would have been allocated to if the segmentation typing tool had been applied and so cannot determine if prioritised segments were indeed those most likely to be 'easy to convert'. Of note, we have included the $12.6 \%$ classified in the 'green' category (and who therefore did not receive the intervention as planned) in the analyses, as no exclusion of men of this type in the no HCD-informed approach is possible.

It was assumed that once trained, every IPC agent would be able to implement the HCD-informed approach as intended. Field observation suggested that implementing the HCD-informed approach requires more education and skills than traditional approaches. It takes time for IPC agents to become familiar with using the tools and, indeed, some IPC agents selected for the trial never became comfortable with using the approach.
Our process evaluation found that younger, male, and better-educated IPC agents appeared more successful at creating demand for VMMC using the approach. Further, as combining the HCD-informed approach and HIVST lengthened the IPC session and resulted in a higher data collection burden, it might not be feasible for the two approaches to be rolled out simultaneously going forward.

Perhaps important is that IPC agents were incentivised according to the national incentive structure (US $\$ 5$ when a boy aged 10-14 years or a man $\geq 30$ years was circumcised and US\$7 when a man aged 15-29 years was circumcised). IPC agents could therefore earn larger amounts by converting groups of younger boys (eg, through schools) with relatively less effort than they could by spending longer time recruiting 'recalcitrant' adult men using the HCD-informed approach.

The power of the trial to show an impact was likely reduced by the fact that relatively few IPC agents ( 75 out of 140 randomised) actually mobilised for VMMC, perhaps reflecting the acceptability and/or feasibility of delivering the interventions. Suboptimal supervision of implementation of the interventions, as indicated by a high number of inactive IPC agents, may have contributed. While the lower than anticipated VMMC conversion rate may have affected the RCT's ability to show an impact, there is no suggestion from the effect sizes of any impact of the HCD-informed approach.

The extent of variability between IPC agents in terms of performance was greater than anticipated. In addition, there was considerable imbalance in characteristics of IPC agents by arm because of the substitution of 20 IPC agents postrandomisation and differential drop out by arm. IPC agents' characteristics were associated with rate of VMMC conversion, and this may have undermined the ability of the trial to demonstrate an impact.

Our trial is one of the first studies to rigorously evaluate an HCD-informed intervention and as delivered in a real-world and resource-constrained setting. Moreover, rigorous procedures were used to ascertain objectively measured outcomes; the RCT was complemented by process evaluation including in-depth qualitative data collection to understand mechanisms of action. However, as with other evaluations of programmatic interventions, our trial was characterised by real life challenges to implementation including a lack of control of the manner in which interventions were implemented. Many of the challenges experienced in this trial are not unique to the interventions tested here, but are common during evaluations of real-world interventions. ${ }^{31}$

In conclusion, the RCT did not show evidence of an effect of interventions on VMMC uptake. That the majority of IPC agents referred fewer than one man a month for VMMC suggests that they may not have been motivated to use these demand creation approaches as designed, which has implications for interventions' feasibility. Importantly, in order to appeal to adult men, other research $^{930}$ has suggested that VMMC demand creation 
interventions should include financial incentives framed as fair compensation. Trial findings will inform future design and implementation of demand creation evaluations.

\section{Author affiliations}

${ }^{1}$ Centre for Sexual Health and HIV/AIDS Research (CeSHHAR) Zimbabwe, Harare, Zimbabwe

${ }^{2}$ Department of International Public Health, Liverpool School of Tropical Medicine, Liverpool, UK

${ }^{3}$ MRC International Statistics and Epidemiology Group, London School of Hygiene \& Tropical Medicine, London, UK

${ }^{4}$ Population Services International, Washington, District of Columbia, USA

${ }^{5}$ Population Services International Zimbabwe, Harare, Zimbabwe

${ }^{6}$ Ministry of Health and Child Care, Harare, Zimbabwe

${ }^{7}$ HIV Department, World Health Organization, Geneve, Switzerland

${ }^{8}$ Department of Clinical Research, London School of Hygiene and Tropical Medicine, London, UK

Twitter Webster Mavhu @webstermavhu, Melissa Neuman @melneuman, Sungai T Chabata @SungaiChabata, Collin Mangenah @cmangenah01 and Cheryl C Johnson@ccasejohn

Acknowledgements The authors would like to thank the VMMC clients and IPC agents who made the study possible.

Contributors WM, FMC and KH led the trial design, with involvement from HAW, $\mathrm{MN}$ and KLF. WM, FMC and MN conceived and designed the study protocol. KH, NT, NM and MM developed the intervention, with support from GN, SX and OM. WM, FMC, SB and CM led the trial implementation, data collection and process evaluation. MN, GM and STC carried out the statistical analysis, with support from HAW and KLF. WM, FMC, MN, KLF, SB and CM led data interpretation with involvement from KH, GM, STC, CM, NT, NM, MM, GN, SX, OM, CJ, ELC and HAW. WM, FMC, MN and KLF wrote the paper and all authors were involved in the review of drafts. All authors have approved the final manuscript.

Funding Bill \& Melinda Gates Foundation; Unitaid as part of the STAR Initiative. Unitaid is a hosted partnership of the World Health Organisation. The funders had no role in study design, data collection, data analysis, data interpretation or writing of the report.

\section{Competing interests None declared.}

Patient consent for publication Not required.

Ethics approval The trial was registered with the Pan African Trial Registry [(registration number PACTR201804003064160]). The protocol was approved by the Medical Research Council of Zimbabwe and Research Council of Zimbabwe (\#2231), Liverpool School of Tropical Medicine (\#17-067) and London School of Hygiene \& Tropical Medicine (\#14460). Qualitative research participants provided written informed consent.

Provenance and peer review Not commissioned; externally peer reviewed.

Data availability statement Data are available upon request. Data from our trial will be made available upon request to the London School of Hygiene \& Tropical Medicine data repository. https://datacompass.Ishtm.ac.uk/.

Supplemental material This content has been supplied by the author(s). It has not been vetted by BMJ Publishing Group Limited (BMJ) and may not have been peer-reviewed. Any opinions or recommendations discussed are solely those of the author(s) and are not endorsed by BMJ. BMJ disclaims all liability and responsibility arising from any reliance placed on the content. Where the content includes any translated material, BMJ does not warrant the accuracy and reliability of the translations (including but not limited to local regulations, clinical guidelines, terminology, drug names and drug dosages), and is not responsible for any error and/or omissions arising from translation and adaptation or otherwise.

Open access This is an open access article distributed under the terms of the Creative Commons Attribution IGO License (CC BY NC 3.0 IGO), which permits use, distribution, and reproduction in any medium, provided the original work is properly cited. In any reproduction of this article there should not be any suggestion that WHO or this article endorse any specific organization or products. The use of the WHO logo is not permitted. This notice should be preserved along with the article's original URL.

Disclaimer: The author is a staff member of the World Health Organization. The author alone is responsible for the views expressed in this publication and they do not necessarily represent the views, decisions or policies of the World Health Organization.

\section{ORCID iDs}

Webster Mavhu http://orcid.org/0000-0003-1881-4398

Melissa Neuman http://orcid.org/0000-0002-8870-6504

Karin Hatzold http://orcid.org/0000-0002-5117-3732

Sungai T Chabata http://orcid.org/0000-0001-7629-1543

Collin Mangenah http://orcid.org/0000-0002-0733-0622

Cheryl C Johnson http://orcid.org/0000-0001-5499-5523

Elizabeth L Corbett http://orcid.org/0000-0002-3552-3181

Helen A Weiss http://orcid.org/0000-0003-3547-7936

Katherine Fielding http://orcid.org/0000-0002-6524-3754

Frances M Cowan http://orcid.org/0000-0003-3087-4422

\section{REFERENCES}

1 UNAIDS. Hiv prevention roadmap 2020: first progress report, 2018. Geneva: UNAIDS, 2018

2 WHO. Preventing HIV through safe voluntary medical male circumcision for adolescent boys and men in generalized HIV epidemics: recommendations and key considerations. Geneva: WHO, 2020.

3 UNAIDS/WHO. Voluntary medical male circumcision: steady progress in the scaleup of VMMC as an HIV prevention intervention in 15 eastern and southern African countries before the SARS-CoV2 pandemic. Geneva: UNAIDS and WHO, 2021.

4 UNAIDS. Global AIDS update: communities at the centre. Geneva: Joint United Nations Programme on HIV/AIDS, 2019.

5 WHO. Voluntary medical male circumcision for HIV prevention: progress brief. Geneva: WHO, 2018.

6 Mavhu W, Hatzold K, Dam KH, et al. Adolescent Wound-Care selfefficacy and practices after voluntary medical male Circumcision-A multicountry assessment. Clin Infect Dis 2018;66:S229-35.

7 Kaufman MR, Smelyanskaya M, Van Lith LM, et al. Adolescent sexual and reproductive health services and implications for the provision of voluntary medical male circumcision: results of a systematic literature review. PLoS One 2016;11:e0149892.

8 Njeuhmeli E, Opuni M, Schnure M, et al. Scaling up voluntary medical male circumcision for human immunodeficiency virus prevention for adolescents and young adult men: a modeling analysis of implementation and impact in selected countries. Clin Infect Dis 2018;66:S166-72.

9 Ensor S, Davies B, Rai T, et al. The effectiveness of demand creation interventions for voluntary male medical circumcision for HIV prevention in sub-Saharan Africa: a mixed methods systematic review. J Int AIDS Soc 2019;22 Suppl 4:e25299.

10 Carrasco MA, Grund JM, Davis SM, et al. Systematic review of the effect of economic compensation and incentives on uptake of voluntary medical male circumcision among men in sub-Saharan Africa. AIDS Care 2018;30:1071-82.

11 Choko AT, Candfield S, Maheswaran $\mathrm{H}$, et al. The effect of demandside financial incentives for increasing linkage into HIV treatment and voluntary medical male circumcision: a systematic review and metaanalysis of randomised controlled trials in low- and middle-income countries. PLoS One 2018;13:e0207263.

12 Thirumurthy $\mathrm{H}$, Masters SH, Rao S, et al. The effects of providing fixed compensation and Lottery-Based rewards on uptake of medical male circumcision in Kenya: a randomized trial. J Acquir Immune Defic Syndr 2016;72 Suppl 4:S309-15.

13 Wambura M, Mahler H, Grund JM, et al. Increasing voluntary medical male circumcision uptake among adult men in Tanzania. AIDS 2017;31:1025-34.

14 Weiss SM, Zulu R, Jones DL, et al. The spear and shield intervention to increase the availability and acceptability of voluntary medical male circumcision in Zambia: a cluster randomised controlled trial. Lancet HIV 2015;2:e181-9.

15 Curran K, Njeuhmeli E, Mirelman A, et al. Voluntary medical male circumcision: strategies for meeting the human resource needs of scale-up in southern and eastern Africa. PLoS Med 2011;8:e1001129.

$16 \mathrm{MOHCC}$. Zimbabwe sustainability transition implementation plan: 2019-2021 - voluntary medical male circumcision. Harare: MOHCC, 2019.

17 Mavhu W, Dauya E, Bandason T, et al. Chronic cough and its association with TB-HIV co-infection: factors affecting help-seeking behaviour in Harare, Zimbabwe. Trop Med Int Health 2010;15:574-9. 
18 Skovdal M, Campbell C, Madanhire C, et al. Masculinity as a barrier to men's use of HIV services in Zimbabwe. Global Health 2011;7:13.

19 Chikovore J, Gillespie N, McGrath N, et al. Men, masculinity, and engagement with treatment as prevention in KwaZulu-Natal, South Africa. AIDS Care 2016;28 Suppl 3:74-82.

20 Hatzold K, Mavhu W, Jasi P, et al. Barriers and motivators to voluntary medical male circumcision uptake among different age groups of men in Zimbabwe: results from a mixed methods study. PLoS One 2014;9:e85051.

21 Skolnik L, Tsui S, Ashengo TA, et al. A cross-sectional study describing motivations and barriers to voluntary medical male circumcision in Lesotho. BMC Public Health 2014;14:1119.

22 Djimeu EW. Scoping report on interventions for increasing the demand for voluntary medical male circumcision. Washington DC: 3ie, 2013.

23 George G, Strauss M, Chirawu P, et al. Barriers and facilitators to the uptake of voluntary medical male circumcision (VMMC) among adolescent boys in KwaZulu-Natal, South Africa. Afr J AIDS Res 2014;13:179-87.

24 Choko AT, Corbett EL, Stallard N, et al. Hiv self-testing alone or with additional interventions, including financial incentives, and linkage to care or prevention among male partners of antenatal care clinic attendees in Malawi: an adaptive multi-arm, multi-stage cluster randomised trial. PLoS Med 2019;16:e1002719.

25 Hatzold K, Gudukeya S, Mutseta MN, et al. HIV self-testing: breaking the barriers to uptake of testing among men and adolescents in
sub-Saharan Africa, experiences from STAR demonstration projects in Malawi, Zambia and Zimbabwe. J Int AIDS Soc 2019;22 Suppl 1:e25244

26 Sgaier SK, Eletskaya M, Engl E, et al. A case study for a psychographic-behavioral segmentation approach for targeted demand generation in voluntary medical male circumcision. Elife 2017;6:6.

27 Gomez A, Loar R, England Kramer A. The impact of market segmentation and social marketing on uptake of preventive programmes: the example of voluntary medical male circumcision. A literature review. Gates Open Res 2018;2:68.

28 Gomez A, Loar R, Kramer AE, et al. Reaching and targeting more effectively: the application of market segmentation to improve HIV prevention programmes. J Int AIDS Soc 2019;22 Suppl 4:e25318.

29 Schulz KF, Altman DG, Moher D, et al. Consort 2010 statement: updated guidelines for reporting parallel group randomised trials. BMJ 2010;340:c332.

30 Kennedy CE, Yeh PT, Atkins K, et al. Economic compensation interventions to increase uptake of voluntary medical male circumcision for HIV prevention: a systematic review and metaanalysis. PLoS One 2020;15:e0227623.

31 Doyle AM, Mulhern E, Rosen J, et al. Challenges and opportunities in evaluating programmes incorporating human-centred design: lessons learnt from the evaluation of adolescents 360. Gates Open Res 2019;3:1472. 